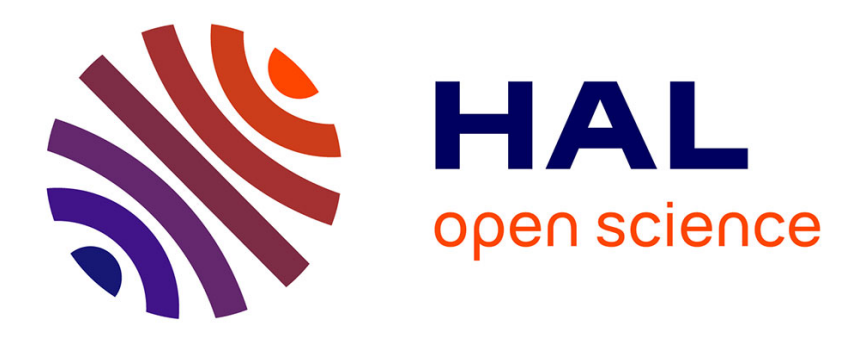

\title{
Le dialogisme dans les relatives disjointes Geneviève Salvan
}

\section{To cite this version:}

Geneviève Salvan. Le dialogisme dans les relatives disjointes. Langue française, 2009, Dialogisme et marqueurs grammaticaux, 163, pp.61-78. 10.3917/lf.163.0061 . hal-01182174

\section{HAL Id: hal-01182174 https://hal.science/hal-01182174}

Submitted on 31 Jul 2015

HAL is a multi-disciplinary open access archive for the deposit and dissemination of scientific research documents, whether they are published or not. The documents may come from teaching and research institutions in France or abroad, or from public or private research centers.
L'archive ouverte pluridisciplinaire HAL, est destinée au dépôt et à la diffusion de documents scientifiques de niveau recherche, publiés ou non, émanant des établissements d'enseignement et de recherche français ou étrangers, des laboratoires publics ou privés. 


\section{LE DIALOGISME DANS LES RELATIVES DISJOINTES}

\section{Geneviève SALVAN}

Armand Colin / Dunod | « Langue française »

$2009 / 3 \mathrm{n}^{\circ} 163 \mid$ pages 61 à 78

ISSN 0023-8368

ISBN 9782200925710

Article disponible en ligne à l'adresse :

http://www.cairn.info/revue-langue-francaise-2009-3-page-61.htm

\section{Pour citer cet article :}

Geneviève SALVAN, «Le dialogisme dans les relatives disjointes », Langue française 2009/3 $\left(n^{\circ} 163\right)$, p. 61-78.

DOI 10.3917/lf.163.0061

Distribution électronique Cairn.info pour Armand Colin / Dunod.

(C) Armand Colin / Dunod. Tous droits réservés pour tous pays.

La reproduction ou représentation de cet article, notamment par photocopie, n'est autorisée que dans les limites des conditions générales d'utilisation du site ou, le cas échéant, des conditions générales de la licence souscrite par votre établissement. Toute autre reproduction ou représentation, en tout ou partie, sous quelque forme et de quelque manière que ce soit, est interdite sauf accord préalable et écrit de l'éditeur, en dehors des cas prévus par la législation en vigueur en France. Il est précisé que son stockage dans une base de données est également interdit. 


\section{Le dialogisme dans les relatives disjointes}

\section{INTRODUCTION : AUTONOMIE ÉNONCIATIVE ET POTENTIALITÉ DIALOGIQUE DES RELATIVES}

L'autonomie énonciative des relatives détachées ${ }^{1}$ a été maintes fois soulignée et mise au compte de leur statut d'assertion indépendante. Comme le montre Haillet (1998 : 217-218) à propos des appositives, ni la négation de l'énoncé enchâssant ni sa mise en interrogation n'affectent l'assertion latérale posée par la relative, comme l'illustre l'exemple suivant :

(1) Jésus vint racheter le genre humain, et triompher du diable, qui nous tente encore. (Voltaire, Dictionnaire philosophique, article "Ange», Paris, Garnier-Flammarion, 2008, p. 40)

$\rightarrow$ Jésus ne vint pas triompher du diable, qui nous tente encore.

$\rightarrow$ Jésus vint-il triompher du diable, qui nous tente encore?

La relative appositive pose une assertion indépendante de l'énoncé enchâssant, et installe dans la phrase un dédoublement énonciatif, qui fait entendre deux instances : "La première est à l'origine de l'énoncé enchâssant la subordonnée relative, la seconde est la source de l'assertion "latérale" enchâssée " (Haillet 1998 : 235). La relative s'accommode de l'insertion de marqueurs dialogiques explicitant la source énonciative lorsque celle-ci diffère de l'énonciateur principal :

$\left(1^{\prime}\right)$ Jésus vint racheter le genre humain, et triompher du diable, qui dit-on/selon $X$ nous tente encore.

En l'absence de marqueur d'altérité, l'assertion est rapportée à l'énonciateur principal $\left(\mathrm{E}_{1}\right)$, ou à une instance collective qui l'inclut: «dans les deux cas,

1. Qu'elles soient explicatives, appositives, enchaînantes ou disjointes, ces relatives sont des constructions détachées du prédicat premier (pour l'opposition détachées vs intégrées, voir Kleiber 1987 ; intégrées vs non intégrées dans une perspective macro syntaxique, voir Gapany 2004 : 77). 
l'assertion latérale posée dans la relative appositive est prise en charge par l'énonciateur principal» (Haillet 1998 : 235). L'hétérogénéité énonciative dans la relative est «masquée » du fait que la relative «dilue l'assertion de $\mathrm{e}_{1}$ dans l'enchâssement qu'en opère $E_{1}$ » (Bres 1998 : 204 et 205) et l'assertion de la relative est régulièrement imputée à un énonciateur $\mathrm{e}_{1}$ coréférant à $\mathrm{E}_{1}$.

En revanche, l'introduction d'un marqueur d'altérité, comme le conditionnel d'altérité énonciative qu'étudie Haillet, rapporte l'assertion à une source distincte de l'énonciateur principal : le dialogisme interdiscursif (reprise d'un dire antérieur d'un tiers), que la relative prévoit en creux, est explicité par le marqueur.

Les relatives disjointes de leur antécédent partagent avec les appositives plusieurs propriétés syntaxiques et énonciatives, notamment le détachement et la capacité à installer dans la phrase un dédoublement énonciatif. Deux caractéristiques les en distinguent cependant : l'éloignement de l'antécédent, séparé du relatif par le groupe verbal, et leur position en fin de phrase (disjonction et extraposition). Elles peuvent être détachées ou non par une virgule:

(2) Trois morts surgirent de l'ombre, dont j'entendis les noms pour la première fois : Robert, Hannah, Simon. (Philippe Grimbert, Un secret, Grasset, 2004, Le Livre de poche, p. 75)

(3) Car aucun guerrier ne foula jamais ma terre ni n'approcha de ma cabane ; car le monde est vaste où se meuvent les guerriers. (Pierre Magnan, Le mystère de Séraphin Monge, p. 317)

Nous défendrons l'idée que les relatives disjointes ne sont pas seulement des variantes positionnelles des relatives appositives et que la disjonction de la relative est un phénomène syntaxique et discursif qui peut recevoir une explication en termes de dialogisation, d'abord parce qu'elle met en jeu des opérations reconnues comme dialogiques: détachement, rhématisation, ensuite parce que l'autonomie énonciative spécifique de ces relatives - en constante négociation avec leur dépendance syntaxique - exploite le potentiel dialogique de leur extraposition. Ce faisant, nous confronterons ces relatives disjointes avec des structures proches et tenterons de faire le départ, sur la base de cette valeur dialogique, entre la relative disjointe et la coordination dont on la rapproche souvent, sur la base de cette valeur dialogique.

\section{LES RELATIVES DISJOINTES : CARACTÉRISTIQUES SYNTAXIQUES ET CORPUS D'ÉTUDE}

La disjonction de l'antécédent est « marquée » en français moderne, la proximité de l'antécédent et du pronom relatif étant la règle, contrairement à ce qui était permis dans la syntaxe classique. Béguelin et al. (2000: 307) appellent « enchaînantes » ces relatives et donnent les exemples suivants :

(4) IBM Suisse a pris l'affaire très au sérieux, qui a prévenu par lettre tous ses clients. (Presse)

(5) Familles en balade et sportifs chevronnés sont désormais comblés, qui peuvent profiter du grand air et de la nature. (Presse) 
Béguelin souligne d'emblée la valeur expressive de telles relatives, ainsi que leur parenté avec les indépendantes: " ces relatives-là, parfois très proches de simples coordinations, produisent à l'écrit un effet de style relativement soutenu » (id.). L'effet stylistique est clairement mis au compte de l'écrit littéraire chez Wagner et Pinchon (1962: 571), qui acceptent l'éloignement du relatif par rapport à l'antécédent comme une "entorse littéraire» à l'usage ${ }^{2}$ : «l'usage veut que la proposition relative suive directement l'antécédent du relatif », mais "dans la langue écrite littéraire la relative peut [toutefois] être séparée de son antécédent par un terme ou un groupe: Alors une vague vint la chercher, qui s'était toujours tenue à quelque distance du village dans une visible réserve. (J. Supervielle)».

Le phénomène est certes fréquent dans l'écrit littéraire, même contemporain :

(6) Alors cet autre bruit s'élève, que d'abord on n'entendait pas tellement il est sourd, d'en dessous, intérieur [...] (Ramuz, Passage du poète)

(7) Barthélemy n'a eu ensuite qu'à s'écarter un peu pour le laisser passer, qui a dit : «Au revoir quand même, et on verra bien. » (Ramuz, La Grande peur)

(8) Monsieur Malbrunot l'enchanta, qui n'avait pas, le brave homme, l'ombre d'un melon d'eau, pas un soupçon de cuisse d'albâtre. (R. Fallet, Le Beaujolais nouveau est arrivé)

(9) Là-dessus, le Glaude bâilla, qui n'avait pas, malgré les racontars, pour habitude de siffler des canons en pleine nuit. (R. Fallet, La Soupe aux choux)

(10) Bonnot s'ennuyait, qui avait des ennuis. (ibid.)

(11) Au bourg, ils se rendirent à la poste, où le receveur s'ébahit, $q u$ 'ils connaissaient de longue date. (ibid. $)^{3}$

sans être pour autant exclu des autres types d'écrit, écrit journalistique comme on l'a vu avec les exemples 1 et 2, mais aussi écrit scientifique (en 12), essai (exemples 13 et 14$)$ :

(12) Le bilan n'est pas pour autant négatif qui traduit sous une forme souvent imagée, toujours concrète, ce qui est de l'ordre de la spéculation. (Christiane Mervaud) $^{4}$

(13) L'enjeu est crucial dans un monde traversé de tensions et de conflits, dont certains touchent précisément aux conséquences du développement technologique. L'opinion souvent se crispe, les citoyens parfois s'entre-déchirent. Des crises éclatent, qui inquiètent. (Étienne Klein) ${ }^{5}$

(14) Il arrive, expliqua-t-il, qu'une nouvelle expérience, ou une nouvelle théorie, soit présentée, qui suggère, sans toutefois l'établir totalement,

2. «Entorse » parfois traitée en termes de figure. Ainsi, P. Bacry (1992: 286) cite une relative disjointe à l'appui de sa définition de l'hyperbate: Quelques braves gens mourraient, dont c'était le métier (M. Yourcenar).

3. Je remercie Catherine Rouayrenc qui m'a signalé ces exemples.

4. « Philosophie et écriture brève : le Dictionnaire philosophique portatif », Revue internationale de philosophie, volume 48, 1994, p. 70.

5. Galilée et les Indiens. Allons-nous liquider la science?, Flammarion, 2008, p. 13. 
l'existence d'un phénomène insoupçonné, lequel est accepté, magnifié, étendu dans ses conséquences, alors que sa base demeure en fait incertaine. (Étienne Klein) ${ }^{6}$

Nous avons choisi un corpus littéraire resserré pour mener ce travail, composé d'un ensemble de romans de Jean Rouaud, de Jean Echenoz et de Pierre Magnan, dont les écritures partagent un goût retrouvé pour la syntaxe classique $^{7}$. Différentes observations peuvent être faites sur ce corpus constitué de quarante exemples :

- du point de vue typographique, l'absence et la présence de pause par la virgule sont également représentées (18 exemples avec virgule, 22 sans virgule) ;

- du point de vue syntaxique, l'antécédent du relatif est le sujet dans 32 exemples, l'objet dans 4 exemples et remplit une autre fonction dans 4 exemples (circonstant ou agent d'une construction absolue $)^{8}$; les relatives sont majoritairement introduites par qui mais pas exclusivement (6 exemples de que, 1 de dont, 1 de où), ce qui atteste la productivité du tour ; la relative est souvent (27 exemples sur 40) en très nette supériorité volumétrique par rapport au groupe formé par l'antécédent et le prédicat verbal.

\section{SYNTAXE ÉNONCIATIVE DES RELATIVES DISJOINTES}

La disjonction cumule une subordination syntaxique et un retard discursif. Le retard de la relative sur la chaîne discursive lui donne un rôle énonciatif particulier : l'assertion posée par la relative est le résultat d'un acte d'énonciation autonome", comme dans le cas d'une relative appositive, mais cette assertion est posée en appui sur l'assertion précédente et la " reconfigure ». Nous le montrerons en examinant successivement la relation sémantique de la relative avec son antécédent et le rôle énonciatif de la relative par rapport à l'assertion principale.

\section{I. Disjonction et structuration de I'information}

Les relatives disjointes, du fait de leur détachement - peu marqué par une virgule dans notre corpus, rappelons-le -, reçoivent dans la plupart des cas

\section{Ibid., p. 65.}

7. Pour Jean Rouaud (13 exemples), il s'agit de : Les Champs d'honneur (1990), Des Hommes illustres (1993), Le Monde à peu près (1996), Pour vos cadeaux (1998), Sur la scène comme au ciel (1999), tous publiés chez Minuit; pour Pierre Magnan (21 exemples) : La Maison assassinée (1985), Le Mystère de Séraphin Monge (1990), La Folie Forcalquier (1995), Un Grison d'Arcadie (1999) publiés chez Denoël ; pour Jean Echenoz (6 exemples) : Courir (2008), Minuit.

8. Nous avons évidemment exclu les relatives compléments d'un verbe de perception ou d'un présentatif (dites parfois " prédicatives » ou " attributives ») parce qu'elles ne sont pas autonomes par rapport au prédicat principal : Je la sentais qui suivait sur mes traits les ombres et les clartés fugitives que les reflets des flammes de l'alambic y faisaient jouer. (P. Magnan, Un Grison d'Arcadie, p. 79)

9. Elle a le statut de «clause autonome» dans l'analyse macro-syntaxique (Béguelin $2000: 310$; Gapany $2004: 76)$. 
une lecture explicative ${ }^{10}$, ce que confirme, entre autres, la possibilité de leur suppression :

(15) Sa cigarette n'est consumée qu'à moitié qui pend à ses lèvres, mais considérant la situation, il lui vient à l'idée sans plus attendre de l'écraser. (J. Rouaud, Les Champs d'honneur, p. 53)

(16) On dégagea l'angle avant du plateau et le lourd dentier fut placé là en soutènement, qui prévenait de sa masse les affaissements à la base. (ibid., p. 91)

Or, il est des cas où la relative disjointe précise un référent énigmatique sans elle. Malgré son apparition retardée et l'intercalation du verbe principal, la relative permet de réévaluer le référent de l'antécédent comme laissé en suspens en son absence ${ }^{11}$. En diachronie d'ailleurs, l'éloignement du relatif pouvait affecter toutes les relatives, quel que soit leur statut sémantique ${ }^{12}$. L'exemple 17 va nous permettre d'illustrer ce fait :

(17) Malgré sa vision tronquée par les œillères, Cinabre contemplait cependant assez d'espace pour en renâcler sourdement.

Je lui promis et longuement que ce soir à Sèderon, la belle avoine l'attendrait dont mon hôtesse aurait soin de le pourvoir avant de me faire fête. (P. Magnan, La Folie Forcalquier, p. 168)

La suppression de la relative, certes possible, conduit à une lecture générique de l'antécédent la belle avoine, lecture impossible avec le partitif, sans doute plus attendu dans ce contexte : « de la belle avoine l'attendrait ». La relative disjointe amène le lecteur à réinterpréter le référent de l'antécédent soit en lecture explicative (on retrouve alors le sens générique de l'article défini et une valeur d'épithète de nature pour belle), soit en lecture déterminative (le cheval aura l'avoine que l'hôtesse prépare à chacune des venues du narrateur, qui a

10. À moins que ce ne soit leur statut d'explicative qui leur permette le détachement... ce que nous ne pensons pas.

11. M. Wilmet (1997 : 194 et suivantes) notait déjà que si les prédicatives (i.e. explicatives) n'exigent pas la nécessité d'une pause, "à l'inverse une pause peut ne pas détruire la valeur déterminative ", comme dans « les cas d'antécédent commun (l'homme qui lutte, qui aime, qui rit...), les cas de retard discursif (le professeur a puni l'élève... qui a triché hier), les cas d'intercalation d'un verbe, représentatifs d'une syntaxe classique qui n'a pas tranché la question de la conjonction du relatif avec son antécédent (Viens, tu fais ton devoir, et le fils dégénère / Qui survit un moment à l'honneur de son père, Cid, II, 2). » Où l'on retrouve nos relatives disjointes... Dans ce cas, s'ajoute au retard discursif un suspens référentiel plus ou moins important, dont la résolution s'effectue dans un second temps.

12. G. Gougenheim dans sa Grammaire de la langue française du XVI siècle (Paris, Picard, 1973 : 96) rappelle que le phénomène est courant : « le pronom relatif peut être fort éloigné de l'antécédent : Son chapeau estoit de soye noire, auquel estoit attachée une riche enseigne (Marguerite de Navarre, Heptaméron, 24 ; éd. M. François, p. 195) ; Le jeune filz s'appelloit Fouquet, de l'age de seize à dix-sept ans, qui estoit bien affaicté et faisoit tousjours quelque chatonnie (Bonaventure des Periers, nouvelle 10, p. 38). M. Huchon (Le français de la Renaissance, Paris, PUF, 1988 : 109) souligne quant à elle que des trois fonctions du relatif (représentant de l'antécédent, rôle syntaxique dans la proposition qu'il introduit, ligature), c'est le rôle de ligature donc d'enchaînement qui est privilégié, à l'imitation du latin. Anne Sancier (Introduction à la langue du XVII siècle, Paris, Nathan, 1993: 124) rappelle enfin que Vaugelas condamnera le tour lorsqu'il fait naître l'ambiguïté, comme dans l'exemple suivant : «Il me faut aussi un cheval pour monter son valet, qui me coûtera bien trente pistoles " (Les Fourberies de Scapin, II, V). 
pris soin de préciser auparavant ce qu'avaient de régulières ces retrouvailles amoureuses).

Les exemples 18 et 19 accentuent la part de la relative dans l'identification référentielle de l'antécédent, dont le référent, malgré l'article défini, reste sinon énigmatique :

(18) Il y avait le lendemain à Oraison un comice agricole où j'avais chance de décrocher ma vingtième médaille pour une bouillie contre le piétin du mouton dont j'étais l'inventeur. Je renonçai à cette fête et à ma recette avec un soupir. J'attelai de bonne heure car la route était longue que je me proposais de parcourir. (ibid., p. 208)

(19) Séraphin regardait avancer lentement le corbillard qui surplombait la foule, car le chemin est raide qui conduit $d u$ moulin à l'église et au cimetière de Lurs. (P. Magnan, La Maison assassinée, p. 273)

En 18, la relative remédie à un défaut d'information sur la route que le lecteur avait néanmoins commencé à combler malgré l'absence d'indication, en restituant les étapes manquantes: le narrateur vient de parler d'un bandit pourchassé, le lecteur en déduit que le renoncement au comice d'Oraison et le choix d'une autre destination sont en lien avec le bandit, que le narrateur veut aller prévenir. En 19, la suppression de la relative produit un énoncé bizarre, et ce d'autant plus que le verbe est au présent et introduit une rupture énonciative. L'identification du référent est parachevée par la relative dont le retard a créé un suspens référentiel (« de quel chemin parle-t-il ?») : elle oblige le lecteur à recalculer un référent qu'il avait dû provisionner par défaut (par exemple «le chemin qu'emprunte le corbillard»).

La tension ressentie à la lecture naît de la divergence entre deux phénomènes anti-orientés, l'étroitesse du lien sémantique de la relative à son antécédent et le retard sur la chaîne discursive de cette dernière. La comparaison avec les énoncés $18^{\prime}$ et 19 'respectant un ordre des mots (plus) attendu :

$\left(18^{\prime}\right)$ J'attelai de bonne heure car la route que je me proposais de parcourir était longue.

$\left(19^{\prime}\right)$ Séraphin regardait avancer lentement le corbillard qui surplombait la foule, car le chemin qui conduit du moulin à l'église et au cimetière de Lurs est raide.

montre que les relatives se retrouvant de fait (ré) intégrées au SN redeviennent déterminatives et perdent leur autonomie énonciative ${ }^{13}$. Le discours est lissé, délivre les informations linéairement, tandis que la relative disjointe les hiérarchise et impose au lecteur un mode de connaissance échelonné du référent, et non « en saisie immédiate».

Une autre différence entre 18, 19 et 18',19' est perceptible, qui a son importance : le changement rythmique par allongement de l'apodose. Cet aspect proprement prosodique de la relative disjointe ne rentre pas précisément dans

13. L'analyse en relative explicative semble bien moins naturelle: j'attelai de bonne heure car la route, que je me proposais de parcourir, était longue; Séraphin regardait avancer lentement le corbillard qui surplombait la foule, car le chemin, qui conduit du moulin à l'église et au cimetière de Lurs, est raide. 
notre propos, mais il est suffisamment prégnant pour l'évoquer même rapidement. À la cadence mineure en $18^{\prime}$ et $19^{\prime}$ correspond la cadence majeure en 18 et 19 qui s'accompagne d'un décrochement intonatif fort : la protase courte car le chemin est raide et l'apodose longue qui conduit du moulin à l'église et au cimetière de Lurs sont alors nettement distinguées par des intonations montante et descendante. L'effet rythmique produit par les relatives disjointes est remarquable et l'analyse stylistique parlerait de "phrase à traîne » : l'acmé de la phrase apparaissant très vite (à raide), l'apodose est amplifiée au détriment de la protase, et la phrase retarde le moment de sa résolution. Ces relatives sont également remarquables par la solution qu'elles apportent à l'exigence de séquence progressive (disposition dans la phrase de masses volumétriques croissantes). Elles permettent au locuteur de "gérer » une expansion nominale très longue par rapport à un prédicat court. Il nous semble même que c'est ce rôle rythmique qui est premier dans bien des exemples que nous avons relevés dans l'écrit non littéraire (voir les exemples 12 et 14). Le choix de la disjonction relève alors souvent de ce compromis "volumétrique » à effectuer, comme c'est encore le cas dans ces deux exemples saisis au fil de nos lectures : «(..) les «corbeaux» le savent bien qui au lieu d'écrire leurs lettres de menace ou de dénonciation à la main, découpent des caractères imprimés pour garder leur anonymat » ${ }^{14} ;$ " (...) l'écriture se fait chorégraphie qui entraîne les phrases dans une sorte de danse autour du sens, une danse qui finit par se transformer en transe ${ }^{15} »$.

Pour revenir à la structure informationnelle des énoncés avec relative disjointe, le retard de la relative en 18 et 19 crée une interrogation sur l'antécédent à laquelle elle répond. La relative est alors bien une assertion autonome, mais une assertion retardée d'autant plus efficace du point de vue pragmatique qu'elle impose à l'interlocuteur de revenir sur du « déjà - malgré tout - identifié ", c'est-à-dire sur un antécédent déjà engagé dans une prédication. Non seulement elle produit un coup de force énonciatif qui oblige à revenir dans un avant du discours, mais encore elle épaissit la relation prédicative. La relative porterait donc moins sur l'antécédent seul que sur une première prédication, ce que nous voudrions maintenant examiner.

\subsection{Promotion du prédicat second et réorientation énonciative}

Les relatives disjointes, du fait de leur détachement, relèvent de la prédication seconde, extérieure au SN et surajoutée à l'énoncé. La rhématisation que subissent les relatives disjointes, à l'instar des appositives, leur donne le statut d'apport informatif second de l'énoncé :

(20) Seul l'uniforme avait changé qui transportait les spectateurs plus de trois siècles en arrière, au temps des chats de Richelieu. (J. Rouaud, Des Hommes illustres, p. 123)

14. Bres et Nowakowska (à paraître).

15. M. Benjelloun "Structure de la phrase et élaboration du sens dans un texte du déluge de J.M.G. Le Clézio », in La phrase littéraire, 2008, Academia-Bruylant, R. Bourkhis et M. Benjelloun dir., p. 151. 
Mais le bras fatiguait à retenir ces masses métalliques hautes de deux mètres cinquante, le sang refluait de la main levée, et fréquemment, comme on visait les mortaises pratiquées dans l'énorme poutre linteau, une parcelle de rouille tombait qui se logeait dans l'ail. (J. Rouaud, Les Champs d'honneur, p. 94)

La même rhématisation s'observerait avec l'intercalation de la relative entre le sujet et le verbe comme en 20', ou avec l'ordre GV + GNS rétablissant la conjonction entre l'antécédent et le pronom relatif comme en 21' :

$\left(20^{\prime}\right)$ Seul l'uniforme, qui transportait les spectateurs plus de trois siècles en arrière, au temps des chats de Richelieu, avait changé.

$\left(21^{\prime}\right) \quad(\ldots)$ et fréquemment, comme on visait les mortaises pratiquées dans l'énorme poutre linteau, tombait une parcelle de rouille (,) qui se logeait dans l'ail.

À la rhématisation commune aux relatives détachées, s'ajoute par la disjonction un effet de rallonge : l'apport informatif de la relative disjointe se réalise plutôt comme report. En 20 et 21, la relative prolonge une phrase qui a les caractéristiques d'un énoncé complet tant syntaxiquement que sémantiquement ${ }^{16}$. Elle se rapproche en cela des divers types d'ajout phrastique abondamment observés et commentés ${ }^{17}$, que la rhétorique appelle « hyperbates ». En 20'et 21', la relative expanse un constituant, tandis qu'en 20 et 21, elle expanse la phrase. Par l'extraposition qu'elle subit, la relative exprime un prédicat second sur un antécédent déjà lesté d'un prédicat premier.

Prenant acte de cette première prédication, la relative est parfois difficile à déplacer dans la phrase, ce qui est le cas en 21 parce que la blessure de l'œil ne peut précéder la chute de la parcelle de rouille, en 22 qui exploite l'opposition entre passé simple et imparfait, et en 23 à cause de la détermination partitive de l'antécédent :

(22) Tout un groupe l'attendait qui l'escorta de sa voiture au magasin. (J. Rouaud, Des Hommes illustres, p. 16) ${ }^{18}$

16. La suppression de la relative en (21) reconfigurerait le rythme de la phrase en une cadence mineure (et un effet de chute, que n'a donc pas choisi l'auteur, malgré le sens de la phrase...).

17. Voir Authier-Revuz 2002, Combettes 2007, Bonhomme 2009.

18. On reconnaît dans cette subordonnée une relative "narrative » de premier plan au passé simple qui s'oppose à une principale d'arrière plan à l'imparfait (Combettes 1992 : 126-127). Cette relative, selon Combettes, peut être transformée en indépendante sans perdre sa fonction de premier plan (126), la principale sert alors de «cadre » à la relative (135). Combettes note également qu'une ponctuation peut détacher principale et subordonnée et souligner la fonction de premier plan de cette dernière, la relative permettant un changement thématique « à moindres frais »: Un carnet relié en cuir de Russie se trouvait là ; que prit Julius et qu'il ouvrit. (Gide, Les caves $d u$ Vatican, cité p. 127). Dans l'approche macro-syntaxique de Gapany (2004: 85), ces relatives qui jouent un rôle dans la progression textuelle sont appelées « relatives de liaison » (ibid.) : elles "se situent "au même niveau" que la clause à laquelle elles sont adjointes. » (Id.) Dans l'économie textuelle, " les propriétés syntaxiques des RDL, dont la relation avec la clause adjacente se réduit à sa plus simple expression, sont exploitées pour produire et souligner tout à la fois une rupture énonciative qui, accompagnée d'un changement de thème, "scande" la progression du texte. » (86). 
(23) J'errais indécis parmi les rayons, feignant d'hésiter sur un titre à choisir parmi les invites chatoyantes des couvertures. Du monde entrait et sortait qui augmentait mon trouble. (P. Magnan, Un Grison d'Arcadie, p. 149-150)

La relative porte moins sur l'antécédent seul que sur l'antécédent déjà engagé dans une relation prédicative : c'est l'entité groupe attendant qui escorte le père du narrateur en 22, c'est l'affluence alternative du monde qui augmente le trouble du narrateur en 23. On peut comparer 22 et 23 à 22' et 23' modifiés :

(22') Tout un groupe l'attendait, et l'escorta de sa voiture au magasin.

(23') J'errais indécis parmi les rayons, feignant d'hésiter sur un titre à choisir parmi les invites chatoyantes des couvertures. Du monde entrait et sortait, et augmentait mon trouble.

En 22', 1'énoncé présente des procès successifs là où en 22 une intentionnalité était perceptible; en 23', c'est la présence du monde qui augmente le trouble du narrateur, alors qu'une paraphrase de 23 ferait apparaître l'expression d'une consécution : «Du monde entrait et sortait et, ce faisant, augmentait mon trouble ».

L'éloignement de la relative, tempéré par sa dépendance syntaxique et le lien anaphorique même faible du relatif ${ }^{19}$, pose ainsi $l^{\prime}$ assertion de la relative en appui sur l'assertion précédente. C'est ce qui justifie que cette structure ne puisse être totalement assimilée à une variante de la coordination. Certes, le rôle d'enchaînement discursif ${ }^{20}$ du relatif (Béguelin 2000 : 307) déporte la structure phrastique de la stricte hypotaxe vers la parataxe et $\mathrm{H}$. Bonnard y reconnaît même le traditionnel « relatif de liaison ${ }^{21}$. Mais alors que la coordination maintient à égalité deux énonciations autonomes, la relative disjointe, tout en se posant comme énonciation autonome, s'articule plus étroitement à l'assertion précédente par le biais de son lien syntaxique avec l'antécédent, déjà modifié par le prédicat principal. Elle construit une représentation complexe hiérarchisée et en général énonciativement orientée là où la coordination opère un cumul informatif. En effet, à la hiérarchisation informationnelle de l'énoncé envisagée plus haut, la relative ajoute une (ré)orientation énonciative

19. Le relatif, rappelle M. Charolles (2007: 194-195), n'a pas la même valeur anaphorique ni les mêmes pouvoirs référentiels que le pronom personnel de troisième personne : même dans les emplois les moins intégrés comme dans la relative disjointe, le relatif «a besoin de s'accrocher à un antécédent (...) qu'il recrute par proximité. (...) Le pronom relatif est une forme liée mémoriellement, il ne réactualise pas, ne réinitialise pas, le référent de son antécédent comme le fait le pronom [personnel] ». M. Charolles conclut que «à proprement parler, le relatif ne réfère pas, il se contente de maintenir son antécédent dans la mémoire des auditeurs/lecteurs ».

20. Cette valeur d'enchaînement est on le sait prépondérante au xvie siècle comme le rappelle M. Huchon (voir note 12) et subsume ses rôles anaphorique et syntaxique.

21. «Le relatif n'est pas forcément neutre, on trouve dans la même fonction [de relatif de liaison] qui, que représentant des noms : Un Cosaque survint, qui prit l'enfant en croupe. (Hugo)» et «qui peut être glosé "et il". » (2001 : 212). Si la coordination est effectivement une glose possible dans l'exemple de Hugo, c'est parce qu'elle trouve dans la succession de deux procès au passé simple un relais favorable (voir ci-dessus la relative narrative). 
du contenu préalablement asserté, pour exprimer une intentionnalité comme en 22, une consécution comme en 21 et 23, ou une restriction comme en 24 :

(24) Nous étions une communauté de gens secrets qui aimaient bien savoir mais non pas informer. Et nous étions tous pareils. Ce qui explique pourquoi le billet de Lucinde pouvait demeurer anodin au fond de mon béret jusqu'à ma mort s'il le fallait. Par ce secret j'avais barre sur tous, que je perdrais sitôt qu'il serait connu. (P. Magnan, Un Grison d'Arcadie, p. 116)

Enfin, certaines relatives disjointes, qui ne sont pas des relatives narratives (voir note 18), s'accommodent mal de la glose en et il, comme les exemples 17, 18,19 et 20 et les suivants :

(25) Il fait semblant d'hésiter cinq minutes mais, comme on l'y encourage, il postule, il est aussitôt admis. L'armée l'avait repéré depuis un moment, qui raffole des athlètes et lui ouvre grand les bras. (J. Echenoz, Courir, p. 32)

(26) Même les médecins s'en mêlent, qui l'ont condamné depuis longtemps sous le prétexte qu'il court en dépit $d u$ bon sens. Ils hochent la tête en pronostiquant que, depuis deux ans, ils s'attendent à le voir expirer à chaque instant. (ibid., p. 56)

(27) Émile démarre comme d'habitude avec sa force mécanique, sa régularité de robot, mais cette fois de façon plus tranquille qu'à Berlin cependant qu'Heino est parti sauvagement, prenant très vite quatre-vingts mètres d'avance. Émile semble s'en désintéresser, qui sait très bien ce qu'il veut faire et qui attend le signal. (ibid., p. 60)

(28) Car crime, cet effondrement brutal d'un homme de quarante et un ans, car il y avait bien un corps étendu sur le sol de linoléum gris de la salle de bains, car notre petite tante Marie, en Miss Marple mystique, arrivée peu après, son chapelet à la main, ne s'y trompa pas, qui connaissait le coupable. (J. Rouaud, Pour vos cadeaux, p. 64)

Le contenu de la relative disjointe est donc promu dans la phrase au rang d'élément rhématisé, mais reporté en bout de chaîne phrastique. Ce rhème second étant retardé, la relative porte sur son antécédent comme ayant déjà fait l'objet d'une prédication. L'exemple (13) que nous rappelons :

(13) Des crises éclatent, qui inquiètent.

nous permet d'illustrer ce fait : ce ne sont pas seulement les crises qui inquiètent, mais les crises quand/du fait/en ceci qu'elles éclatent. Alors que l'énonciation indépendante des relatives appositives rappelée dans la première partie était mise en évidence par la décomposition suivante ${ }^{22}$ :

(13') Des crises, qui inquiètent, éclatent.

$\rightarrow$ Des crises éclatent.

$\rightarrow$ Des crises inquiètent.

Les énoncés avec relatives disjointes se verront quant à eux décomposés plutôt ainsi :

22. Décomposition que rappelle P.P. Haillet, 1998 : 214. 
(13) Des crises éclatent, qui inquiètent.

$\rightarrow$ Des crises éclatent.

$\rightarrow$ Des crises éclatant/l'éclatement des crises inquiète (nt).

La relative disjointe oblige l'interlocuteur à récupérer ce qui a déjà été dit de l'antécédent, pour le réintégrer dans une seconde prédication. La récupération est parfois explicitée par une continuité isotopique comme en 16, en 29 et $30^{23}$ :

(16) On dégagea l'angle avant du plateau et le lourd dentier fut placé là en soutènement, qui prévenait de sa masse les affaissements à la base. (J. Rouaud, Les Champs d'honneur, p. 91)

(29) Certains ont un sort plus enviable, qui, souverainement indifférents, se prélassent sur la margelle d'un puits ou se livrent sur le rebord d'une fenêtre à une interminable toilette. (J. Rouaud, Des Hommes illustres, p. 32)

(30) Le procès-verbal du maire n'avait en rien refroidi son ardeur, au contraire : il s'efforçait d'uriner plusieurs fois par jour et la colère lui revenait à chaque miction nouvelle, qu'il exprimait par des imprécations et des injures. (Pierre Magnan, La Naine, p. 87)

Le prédicat de l'assertion principale, en 16, placé en soutènement, est rappelé par le prédicat prévenir de sa masse ; en 29, avoir un sort plus enviable est explicité par se prélassent, interminable toilette; en 30, le retour de la colère est prolongé par imprécations, injures, tous rappels qui montrent que les relatives se construisent en prenant en charge le prédicat principal préalablement associé à son antécédent.

Si les paraphrases précédentes sont acceptables, on peut conclure que la relative opère une réévaluation de l'assertion principale par promotion du prédicat second, ce qui la distingue de l'énonciation parenthétique de la relative appositive (Kleiber 1987), qu'elle suive l'antécédent ou qu'elle soit anticipée comme dans l'exemple suivant ${ }^{24}$ :

(31) [Les curistes] pouvaient voir de là-haut, qui accrochait sur des tessons les lueurs des grands feux, le vase à géraniums renversé et la terre noire autour qui maculait les fleurs rouges et qui jurait avec le blanc du pavillon. (P. Magnan, La Folie Forcalquier, p. 352)

La position finale de la relative disjointe lui confère un rôle discursif d'hyperbate plus que de parenthèse : l'énonciation « hyperbatique » de la relative disjointe se saisit sur l'axe syntagmatique du discours, contrairement à l'énonciation parenthétique de l'appositive de nature paradigmatique (d'où son point d'insertion variable). Son rôle d'enchaînement énonciatif est alors

23. Voir aussi la continuité isotopique dans l'exemple cité plus haut (p. 65) : l'écriture se fait chorégraphie qui entraîne les phrases dans une sorte de danse autour du sens, une danse qui finit par se transformer en transe.

24. Gapany (2004: 74) parle de « relatives mobiles » et constate que "l'ordre des mots appliqué dans ce type de structures suggère que la relative doit être analysée sur le modèle des insertions parenthétiques, dont le placement à l'intérieur d'une séquence discursive est assez peu contraint ». 
très net dans la mesure où elle ne juxtapose ni n'empile les énonciations mais les articule, la seconde venant gloser, expliciter, renforcer, justifier ou amender la première. C'est la prise en compte du déjà asserté et la réorientation énonciative qu'elle en effectue qui nous semblent être au cœur du dialogisme de ces relatives.

\section{LE DIALOGISME DANS LES RELATIVES DISJOINTES}

La relative disjointe porte sur du préalablement asserté et son énonciation s'effectue en appui sur l'énoncé précédent, pour construire progressivement un objet de discours (exemples 17, 18 et 19) ou pour réorienter ce qui vient d'être posé (exemples 22, 23 et 24). Le détachement à droite de la relative fragmente la phrase, donne à la relative son autonomie et accentue l'hétérogénéité énonciative.

$C^{\prime}$ est donc sur un énoncé déjà actualisé que porte la relative, ce qui n'est pas sans rappeler ce que disent Bres \& Nowakowska (2006 : 29) de l'énoncé dialogique: «dans l'énoncé monologique l'actualisation déictique et modale porte sur un dictum, dans l'énoncé dialogique, cette opération s'effectue non sur un dictum, mais sur (ce qui est présenté comme) un énoncé déjà actualisé. (...) L'énoncé dialogique présente donc une dualité énonciative hiérarchisée ». Bien entendu, la relative ne reprend/cite/rapporte pas l'énoncé précédent mais dans la dynamique de la phrase, elle le rappelle et dialogue avec lui, ce que ne fait pas la relative appositive.

L.-F. Céline, dans les quelques phrases en exergue au Voyage au bout de la nuit, fournit une relative disjointe assez exemplaire :

(32) C'est un roman, rien qu'une histoire fictive. Littré le dit, qui ne se trompe jamais.

L'assertion posée par la relative porte sur une prédication déjà réalisée : son énonciation est non seulement indépendante mais aussi rétroactive, et procède à une validation, problématique ici, de l'assertion principale. La phrase fait entendre deux discours qui entrent en interaction sur le même objet: Littré le dit $^{25}$ et Littré disant - c'est-à-dire cité comme garant, comme auctoritas - ne se trompe jamais et deux voix, que signale l'intonation descendante d'une telle relative. L'éloignement de la relative permet de dire en deux mouvements énonciatifs successifs deux assertions sur l'objet, le relatif maintenant un lien. Le décrochage énonciatif dont le relatif est l'indice affecte ici :

- le même énonciateur à deux stades différents de son énonciation, soit en commentaire sur son propre discours (autodialogisme), soit en travail sur la compréhension-réponse de l'allocutaire (dialogisme interlocutif). En effet, l'énonciateur, convoquant l'autorité de Littré (Littré le dit), anticipe sur l'opinion avantageuse généralement partagée, la convoque même si c'est pour s'en moquer.

25. Le pronom le est anaphorique de l'énoncé « un roman est une histoire fictive ». 
- des énonciateurs différents, parce que la relative fait entendre ici dialogiquement un autre énonciateur (dialogisme interdiscursif): un énonciateur enchâssé $e_{1}$, disons conformiste, distinct de l'énonciateur enchâssant, que l'on savait anti-conformiste, et auquel ce dernier impute ironiquement l'assertion de la relative. L'ironie est d'autant plus vive que l'énonciateur $E_{1}$ peut prendre en charge l'assertion posée par la relative mais en la rapportant au on de la doxa (actualisant en même temps : $E_{1}$ inclus dans $\mathrm{e}_{1}$, mais $\mathrm{E}_{1}$ distinct de $\mathrm{e}_{1}$ ).

Si le dédoublement énonciatif est identique à celui d'une relative apposée à l'antécédent (Littré, qui ne se trompe jamais, le dit), l'effet discursif n'est en revanche pas le même: l'énonciation de la relative disjointe s'exhibe comme greffée sur un énoncé déjà proféré et réorienté (ici pour appuyer l'effet ironique).

Les trois dimensions du dialogisme sont intéressées en discours par ces relatives disjointes, selon le jeu des interactions contextuelles, que nous allons maintenant préciser en les séparant pour l'analyse, même si évidemment les énoncés peuvent actualiser en même temps plusieurs types de dialogisme.

\section{I. Le dialogisme interlocutif dans les relatives disjointes}

La relative disjointe apparaît nettement tournée vers le destinataire lorsqu'elle anticipe une question de sa part, prévient ses demandes d'éclaircissements, précise ou corrige par avance son interprétation. Reprenons l'exemple 18:

(18) Il y avait le lendemain à Oraison un comice agricole où j'avais chance de décrocher ma vingtième médaille pour une bouillie contre le piétin du mouton dont j'étais l'inventeur. Je renonçai à cette fête et à ma recette avec un soupir. J'attelai de bonne heure car la route était longue que je me proposais de parcourir.

L'antécédent la route était pleinement identifié dans l'esprit du narrateurénonciateur, qui s'apercevant qu'il n'en a peut-être pas assez dit, complète à destination de son lecteur la référence. Cette relative peut alors être analysée en terme de dialogisme interlocutif sous sa forme responsive, qui anticipe sur un énoncé enchâssé [e], attribué à un énonciateur $\mathrm{e}_{1}$ correspondant au lecteur (« de quelle longue route parlez-vous ? ») et répond par avance à cette question.

C'est ce dialogisme interlocutif qui nous paraît à l'œuvre de manière privilégiée, dans les relatives disjointes qui retravaillent le référent de l'antécédent. Ainsi dans l'exemple 19:

(19) Séraphin regardait avancer lentement le corbillard qui surplombait la foule, car le chemin est raide qui conduit du moulin à l'église et au cimetière de Lurs.

Au décrochage énonciatif opéré par l'explication au présent (car le chemin est raide) premier indice de dialogisme interlocutif anticipatif, s'ajoute un deuxième décrochage, venant répondre à un énoncé $[\mathrm{e}]$ absent, imputé au lecteur et anticipé par le narrateur: "de quel chemin raide parlez-vous?». La 
relative disjointe répond par anticipation à une question que le lecteur se pose sur le référent de l'antécédent. Le suspens informatif est créé par la disjonction, et résolu dialogiquement par la relative ${ }^{26}$.

Parfois, sans anticiper nécessairement sur une question du lecteur, la relative peut jouer sur la représentation du fait raconté et prévenir une inférence que le lecteur pourrait tirer de la première assertion (en 33 « un cadavre sent bon $»)$ :

(33) Sauf ma grand-mère sur son lit de mort (...), je n'avais jamais vu de cadavre. Celui-ci était propre et net, et même un léger parfum de lotion flottait autour de lui que commençait à dominer l'odeur fécale du corps inerte et sans défense contre la physiologie du décès. (P. Magnan, Un grison d'Arcadie, p. 17)

La relative accueille la voix d'un énonciateur qui ne remet pas en cause le point de vue énoncé dans la principale mais qui en limite la portée. L'énonciateur $\mathrm{e}_{1}$ à l'origine de la relative peut être distinct du narrateur, ce qu'attestent les choix lexicaux techniques et soutenus de "odeur fécale ", "physiologie du décès ", hétérogènes par rapport à l'idiolecte du narrateur, ou se superposer à $\mathrm{E}_{1}$ pour s'en distancier.

\subsection{L'autodialogisme dans les relatives disjointes}

Une autre dimension dialogique actualisée par ces relatives est celle du dialogisme intralocutif, ou autodialogisme, de l'énonciateur-narrateur en train de dialoguer avec sa propre énonciation : « le locuteur est son premier interlocuteur dans le processus d'autoréception : la production de sa parole se fait constamment en interaction avec ce qu'il a dit antérieurement, avec ce qu'il est en train de dire, et avec ce qu'il a à dire » (Bres 2005 : 53). L'énonciateur dialogue avec le discours qu'il a tenu pour le préciser, souvent pour renforcer ou justifier l'assertion principale (ici en 29 et 36), valider un choix lexical (« en otage » en $34)$, justifier un fait (en 35):

(29) Certains ont un sort plus enviable, qui, souverainement indifférents, se prélassent sur la margelle d'un puits ou se livrent sur le rebord d'une fenêtre à une interminable toilette. (ibid., p. 32)

(34) Il y avait parmi eux des hommes qui aimaient les arbres. J'en ai vu un ou deux qui pleuraient presque. Ils nous laissaient le géologue en otage, qui ne reprendrait le car que le lendemain. (Pierre Magnan, Le Mystère de Séraphin Monge, 1990, folio policier, p. 54)

(35) Du coup certains s'enhardirent qui considéraient sous cet éclairage nouveau que la réputation $d u$ grand Joseph était somme toute surfaite. (J. Rouaud, Des Hommes illustres, p. 20)

(36) Quoique certains visiblement ne semblent pas rebutés, qui sortent $d u$ terrain plus sales qu'un vainqueur de Paris-Roubaix - les années pluvieuses, les seules qui comptent dans la légende de l’Enfer du Nord -,

26. En termes praxématiques, la relative est un lieu de "passage » d'une subjectivité en idem (qui suppose la non-disjonction du locuteur d'avec l'autre et donc l'absence de sa prise en compte) à une subjectivité en ipse (où la disjonction existe et impose l'élucidation du sens pour l'autre). 
la couleur du maillot même plus discernable, au point qu'on se demande comment ils font en entrant dans les vestiaires coupés en deux (d'un côté les autochtones, de l'autre les visiteurs) pour ne pas se tromper de porte. (J. Rouaud, Le Monde à peu près, p. 16)

Dans ces exemples, l'énonciateur principal $\mathrm{E}_{1}$ prend à son compte l'assertion de la relative et s'appuie sur elle pour renforcer l'assertion précédente, et même pour délivrer l'essentiel du propos (la principale se donnant presque pour anticipatrice : «je dis «sort plus enviable» parce que «se prélassent »; «je dis «pas rebutés » parce que «sortent du terrain plus sales...»).

\subsection{Le jeu des dimensions dialogiques}

Les dimensions dialogiques sont parfois si intimement liées qu'il est difficile d'en faire le départ. Elles jouent à différents niveaux dans l'énoncé. Dans l'exemple 34, l'expression « en otage » apparaît dans un énoncé qu'on peut interpréter comme du discours indirect libre (DIL) faisant entendre la voix du narrateur et celle des experts venus examiner les glissements de terrains dans la forêt. Ce que réalise la relative, c'est un retour sur l'énonciation, sur une manière de dire hétérogène, pour l'expliciter et/ou la justifier. Même si on conteste la lecture en DIL, la relative revient sur du déjà dit et conduit à relire « en otage » comme une combinaison d'usage et de mention caractéristique de la modalisation autonymique. L'expression "en otage » en effet surprend dans la narration et se donne comme emprunt au discours d'un tiers. Si la phrase s'arrêtait là, elle serait achevée syntaxiquement, mais constituerait une assertion bizarre, au regard de ce que le lecteur sait et de la situation et du narrateur. Que fait alors la relative? Elle est orientée dialogiquement vers l'amont de la phrase : elle pointe l'incongruité du choix lexical de « en otage » (dialogisme interdiscursif), elle en justifie parallèlement l'énonciation (dialogisme interlocutif) et dépasse finalement la dissonance qui pourrait se faire entendre (autodialogisme).

Le jeu dialogique peut également être mis en évidence par la confrontation de ces relatives disjointes avec les phrases non dépendantes juxtaposées ou coordonnées dont on les rapproche :

(35) Du coup certains s'enhardirent qui considéraient sous cet éclairage nouveau que la réputation du grand Joseph était somme toute surfaite. (Jean Rouaud, Des Hommes illustres, p. 20)

(37) Mais pour grand-mère c'est du pareil au même, qui ne comprend pas qu'on n'ait pas encore dynamité le Massif central et coupé tout droit. (Jean Rouaud, Les Champs d'honneur, p. 44)

(28) Car crime, cet effondrement brutal d'un homme de quarante et un ans, car il y avait bien un corps étendu sur le sol de linoléum gris de la salle de bains, car notre petite tante Marie, en Miss Marple mystique, arrivée peu après, son chapelet à la main, ne s'y trompa pas, qui connaissait le coupable. (Jean Rouaud, Pour vos cadeaux, p. 64)

Les relatives paraissent en effet ici proches de simples assertions juxtaposées :

(35') Certains s'enhardirent, ils considéraient sous cet éclairage nouveau que la réputation du grand Joseph était somme tout surfaite 
(37') Pour grand-mère c'est du pareil au même, elle ne comprend pas qu'on n'ait pas encore dynamité le Massif central

$\left(28^{\prime}\right) \quad(.$.$) car notre petite tante Marie, en Miss Marple mystique, arrivée peu$ après, son chapelet à la main, ne s'y trompa pas, elle connaissait le coupable.

Il nous semble néanmoins qu'il y a une différence énonciative entre ces structures : dans les exemples remaniés $35^{\prime}, 37^{\prime}$ et $28^{\prime}$, les énoncés juxtaposés sont susceptibles d'une lecture en DIL, donc en dialogisme interdiscursif, lecture favorisée contextuellement par la rupture passé simple/imparfait en 35'et $28^{\prime}$, et le marqueur dialogique “pour grand-mère » en 37'. Dans les exemples originaux, la disjonction de la relative enrichit l'interprétation et reconfigure l'énonciation, en intégrant une dimension dialogique interlocutive responsive qui prévient une interrogation que pourrait formuler le lecteur ("pourquoi dites-vous cela ? ») et autodialogique (processus de validation par le narrateur de ce qu'il vient de dire). Le maintien d'une structure de dépendance syntaxique équivaut au maintien d'un contrôle énonciatif plus fort de $\mathrm{E}_{1}$ et donc à un dialogisme contrôlé tout en étant exhibé.

La relative peut également exploiter le décrochage énonciatif entre discours et récit. Dans l'exemple 38, la principale en régime de discours, prise en charge par le narrateur, est suivie d'une relative en régime de récit. Celle-ci efface énonciativement le narrateur et fait émerger grâce à l'expression « entacher sa réputation » un dialogisme interdiscursif qui fait entendre un énonciateur $\mathrm{e}_{1}$, le directeur de l'établissement :

(38) Dix enfants nouveau-nés succomberont, que le directeur de l'établissement préféra sacrifier plutôt que de risquer, en annonçant la terrible nouvelle, d'entacher sa réputation. (J. Rouaud, Pour vos cadeaux, p. 80)

La valeur dialogique de la relative disjointe naît de son interaction avec l'assertion principale, soit qu'elle la confirme et la prenne en charge, soit qu'elle l'explicite ou la justifie, soit au contraire qu'elle en limite la portée. Il nous semble que c'est cette interaction entre énonciations qui distingue les relatives disjointes des explicatives qui se présentent comme des assertions indépendantes de - mais aussi relativement "indifférentes » à - l'assertion enchâssante.

\section{CONCLUSION}

Dans le champ dialogique de la subordination, les relatives disjointes jouent leur partie: relevant du dédoublement énonciatif déjà mis en évidence à propos des relatives appositives et partant, de toute relative détachée, elles ajoutent, du fait de leur position, une réorientation énonciative que les relatives appositives ne prévoyaient pas nécessairement. La notion de dialogisme et les avancées récentes qu'elle a connues (Bres et Nowakowska 2008) permet de mieux les décrire et d'appréhender leur spécificité énonciative dans l'exploitation subtile des trois dimensions dialogiques, interdiscursive, interlocutive et intralocutive. 
Les relatives éloignées de leur antécédent témoignent du fait que l'ordre des mots n'est pas seulement soumis aux contraintes de dépendance et de clarté des rapports syntaxiques mais répond aussi à des déterminations énonciatives et cognitives. Au regard de la norme syntaxique, ces relatives se rapprochent $\mathrm{du}$ fonctionnement subversif des hyperbates qui « distord [ent] la grammaticalité normative de la phrase » et projettent «en avant sur l'ordre séquentiel de l'énoncé » un constituant ${ }^{27}$. Mais au regard de la dynamique énonciative, ces relatives témoignent du dialogue interne du discours, d'un dire en construction soumis à l'interaction permanente avec les discours autres et à l'ajustement intersubjectif ${ }^{28}$.

\section{Références bibliographiques}

Authier-ReVuz J. et Lala M.-C. (éds), 2002, Les figures d'ajout. Phrase, texte, écriture, Paris, PUPS.

BACRY P., 1992, Les figures de style, Paris, Belin.

BÉGUELIN M.-J. (dir.), 2000, De la phrase aux énoncés: grammaire scolaire et descriptions linguistiques,

Bruxelles, De Boeck-Duculot.

BonHOMme M., 2005, Pragmatique des figures du discours, Paris, Honoré Champion.

BONHOMME M., 2009, «Entre grammaire et rhétorique. L'hyperbate comme extraposition problématique », Les Linguistiques du détachement, Actes du colloque international de Nancy (7-

9 juin 2006), D. Apothéloz, B. Combettes, F. Neveu (éds), Bern, Peter Lang, I 17 - 128.

BONNARD H., 200I, Les trois logiques de la grammaire française, Bruxelles, Duculot.

BRES J. 1998, «Entendre des voix: de quelques marqueurs dialogiques en français », in Bres J.,

Delamotte-Legrand R., Madray-Lesigne F., Siblot P. (éds), L'autre en discours, Montpellier III, Praxiling, $191-212$.

BRES J., 2005, « Savoir de quoi on parle : dialogal, dialogique, polyphonique », in Bres et al., p. 47-62.

BRES J., 2007, «Sous la surface textuelle, la profondeur énonciative. Les formes du dialogisme de l'énoncé », in R. Therkelsen, N. Møller Andersen et H. Nølke (éds), Sproglog Polyfoni, Aarhus Universitetsforlag, 37-54.

BRES J., NOWAKOWSKA A., 2006, « Dialogisme : du principe à la matérialité discursive », in Le sens et ses voix. Dialogisme et polyphonie en langue et en discours, Recherches linguistiques $n^{\circ} 28$, Université Paul Verlaine, Metz, 2I-48.

BRES J., NOWAKOWSKA A., 2008, « J'exagère !... Du dialogisme interlocutif », in M. Birkelund, M.-B. Mosagaard Hansen et C. Norén (éds), L'énonciation dans tous ses états, Bruxelles, Peter Lang, I-27.

BReS J., Haillet P., Mellet S., Nølke H., Rosier L. (éds), 2005, Dialogisme et polyphonie: approches linguistiques, Actes du colloque de Cerisy, Bruxelles, Duculot.

CHAROLLES M., 2007, « Comment évaluer les effets des relatives en qui sur les chaînes de référence ? », Parcours de la phrase. Mélanges offerts à Pierre Le Goffic, textes réunis par M. Charolles, N. Fournier,

C. Fuchs et F. Lefeuvre, Paris, Ophrys, 193-212.

Combettes B., 1992, L'Organisation du texte, Metz, Université de Metz.

27. Bonhomme (2005 : 33). L'effet figural de l'hyperbate est selon Bonhomme de promouvoir une vision dissociative de l'énonciateur sur la réalité exprimée et une prise de position «emphatique » (Bonhomme : 41).

28. À cause du corpus que nous avons choisi, nous n'avons cessé de croiser au cours de notre étude des questions plus stylistiques liées à l'énonciation romanesque et à la construction de l'ethos du narrateur. Nous ne pouvions matériellement pas aborder cette question dans les limites " grammaticales et dialogiques » de ce numéro et projetons de le faire dans un article à venir. 
Combettes B., 2007, «Les ajouts après le point: aspects syntaxiques et textuels », Parcours de la phrase. Mélanges offerts à Pierre Le Goffic, | 19-131.

FUCHS C. (dir.), 1987, Les types de relatives, Langages 88, Paris.

GAPANY J., 2004, Formes et fonctions des relatives en français, Berne, Peter Lang.

HAILlET P.P., 1998, «Quand un énoncé en cache un autre: le conditionnel et les relatives appositives », in J. Bres et al. (éds) L'autre en discours, 213-238.

KLEIBER G., 1987, «Relatives restrictives et relatives appositives » : une opposition introuvable?, Tübingen, Max Niemayer Verlag.

LE GOFFIC P., 1979, «Propositions relatives, identification et ambiguïté, ou : Pour en finir avec les deux types de relatives », DRLAV n² I, Université de Paris VIII, Vincennes, I35-145.

WAGNER R. L. et PINCHON J., 1962, Grammaire du français classique et moderne, Paris, Hachette.

WILMET M., 1997, Grammaire critique du français, Paris, Hachette. 